\title{
Labyrinthe
}

19 | 2004 (3)

Le Bel Aujourd'hui

\section{Chroniques de la fin du monde}

Laurent Dubreuil

\section{OpenEdition}

Journals

Édition électronique

URL : http://journals.openedition.org/labyrinthe/236

DOI : $10.4000 /$ labyrinthe.236

ISSN : 1950-6031

Éditeur

Hermann

Édition imprimée

Date de publication : 15 décembre 2004

Pagination : 31-42

Référence électronique

Laurent Dubreuil, «Chroniques de la fin du monde », Labyrinthe [En ligne], 19 | 2004 (3), mis en ligne le 18 juin 2008, consulté le 10 décembre 2020. URL : http://journals.openedition.org/labyrinthe/236 ;

DOI : https://doi.org/10.4000/labyrinthe.236

Propriété intellectuelle 


\title{
CHRONIQUES \\ DE LA FIN DU MONDE
}

\author{
Laurent DuBREUIL \\ laurentdubreuil@wanadoo.fr
}

\section{Certes - mais}

Elle nous est promise depuis si longtemps, prédite par tant de bouches, prévue par des regards obscurcis ou clairs, comment y croire encore, une fois encore du moins? Oui, la fin du monde maintenant. Nous ne serions pas les premiers à le proclamer; l'abondance d'ouvrages compilateurs parus dans les années qui restaient au siècle précédent nous l'ont rappelé avec entêtement: les formes de la fin ne manquent pas, de l'Antiquité (babylonienne, hébraïque, même grecque) jusqu'à nous. Mais compte plutôt la question: serions-nous les derniers à marquer la fin? Et si le monde périt, bien sûr, nous nous éteindrons avec lui - quitte à lui survivre ailleurs. Dans l'épaisseur des doctrines terminales figurent ces stoïciens qui croyaient apparemment à la palingénésie du tout, une métempsycose de l'univers, poursuivi dans un nouvel avatar ${ }^{1}$. L'astronome, éditeur, science-fictionneur Camille Flammarion affirmait aussi en 1894 que les humains périraient un jour avec leur Terre, mais qu'ils se réincarneraient dans une civilisation supérieure, par exemple sur Jupiter ${ }^{2}$. La fin du monde nous renvoie aussi bien à ce que nous nommons notre monde, et à ses au-delà, pas forcément meilleurs. Et si l'on veut penser la chose (mais le sujet fait peur, il provoque le rire ou le retrait), il s'agit de rappeler cela, de ne pas s'arrêter aux formes, aux types. Il se pourrait qu'une fin du monde se dise «par-delà le miroir » des paroles prêtes à l'emploi, des simples traditions.

Maintenant que s'évacue un certain millénarisme pour la montre, qu'est liquidée la grande peur arachnoïde du bug de l'an deux fois mille,

\footnotetext{
1. Stoicorum veterum fragmenta, vol. II, fragment 624.

2. La Fin du monde, p. 340 ; voir sur le sujet les révélations de « l'ombre » du pharaon Khéops, p. 356.
} 
cette terreur commandée qui ne prit pas, qu'est dispersée la mode des publications «sérieuses» sur le phénomène, nous touchons un bon moment pour réfléchir. Il faudrait plus d'étapes qu'il n'en sera compté cette fois. Prenez les interruptions entre les étapes de cet article pour une véritable discontinuité, pour des pauses plus qu'une pose. La fin sera-t-elle jamais délivrée dans une version toute cohérente, organisée, rationalisée? Reportons cela à plus tard, à l'après de l'après. Il n'est peut-être simplement pas encore temps de circonscrire jusqu'au bout.

\section{Words}

Les paroles se déposeraient. Il n'y aurait alors plus qu'à faire l'étude stratigraphique des sédiments. Des ouvrages d'intérêts divers se livrent à l'exploration chronologique. Ils mélangent les discours pour mieux les départager et babiller là-dessus. Ils recensent des répétitions, ils remarquent des filières, ils font surgir le collectif dans le langage. Comme un talisman, ils agitent une certaine ironie. L'humour et le détachement sont ainsi célébrés en quatrième de couverture de deux livres disponibles en français, La Fin $d u$ monde de Lucian Boia et Apocalypses et millénaires d'Eugen Weber. Le ton plaisant a le don de trancher avec la noirceur des prédictions rapportées. On s'amuse. Pour le savant, c'est le fun de la fin. Weber ne cache pas en préambule qu'il a cherché un divertissement dans un domaine qui se rapporte à ses autres recherches (l'Action française, la Fin-de-siècle) mais qui lui échappe largement. Il se place dans un relativisme distanciant. Les révélations ne s'avérant guère, les deux historiens en déduisent qu'elles n'ont qu'une valeur symbolique. Elles deviennent les scansions répétitives des civilisations d'hommes mortels, elles forment une «histoire sans fin» (Boia). Les différentes valeurs que prennent les mots de la fin sont du coup annulées, au profit d'une illusion sous-jacente et unitaire. Eh pardi ! en restant au niveau des réifications, on ne s'inquiète pas trop. Il est indispensable de repérer des invariances, voire de proposer une typologie, ainsi que le font Boia ou Weber. Encore faudrait-il aussitôt voir comment certaines pensées mobilisent ces figures et s'élaborent malgré elles. Les histoires récentes dont nous disposons souffrent de deux grands défauts. Non seulement elles réduisent le langage (soit la pensée, dans un côté linguistic turn) à ses codes seconds (genres, grammaire des formes...), mais elles peinent à identifier 
correctement leurs propres objets (elles les appréhendent mal intellectuellement, et elles font d'étonnantes impasses).

Les lieux communs de la fin du monde ne sont pas des données pures. Il est néanmoins possible de les agencer sous des traits simplifiés et construits par l'interprète (dans tous les sens du mot). On a le droit, comme ce chercheur italien, de parler du mixing new age, qui caractériserait l'éclectisme post-moderne des prophètes (Paco Rabanne, l'ordre du Temple solaire) $)^{3}$. Encore se rappellera-t-on que le mélange n'est pas d'hier, ce que ne manquent jamais de dire, paradoxalement, ceux qui remarquent l'antique hybridation entre pistes dites assyriennes et visions nommées grecques ${ }^{4}$. L'utile simplification n'est pas un constat objectif du travail temporel des dogmes. Chaque parole terminale la refait pour son propos, avec ce qui lui préexistait. Reconnaissons donc des groupes d'énoncés qui se perpétuent, tout en mettant en doute leur existence séparée. Admettons même que, souvent, les phrases se figent en des postures schématiques, qui ressemblent fort aux contre-images délivrées par l'histoire. Ne nous arrêtons pas à elles ; considérons plutôt la vivacité que les clichés seuls, afin de mieux comprendre comment autre chose se dit par instants qu'un bégaiement d'oracles vieillis. Voici.

Dans l'ensemble du corpus, l'apocalypse nous encombre, et avec elle cet apocalyptisme qui dispose aujourd'hui d'une encyclopédie, pas mauvaise au demeurant ${ }^{5}$. Euvre de synthèse dans son écriture canonique, la Révélation biblique est victime de sa propre fortune. Si les mots de l'apocalypse signifient pour nous sans forcément se solidifier dans des sens préconçus, le dernier livre du Livre a également engendré l'imagerie, qu'on aurait tort (derechef) de croire alpha et oméga de la question - mais également tort de biffer d'un trait parce qu'elle est dorénavant trop rudimentaire. Si le chanteur Marilyn Manson eut quelque opinion valable sur le sujet ${ }^{6}$, il reste qu'en général son appel à l'Antéchrist sert d'abord une imagerie. À son usage personnel, il compose un recueil grotesque ${ }^{7}$ de clichés, qui se veut l'image déformée d'une société à la veille de la destruction. L'apocalypse est une

\footnotetext{
3. Giovanni Firolamo, Millenario e New Age, ch. 8.

4. Voir l'insistance scolaire de Christine Dumas-Reungoat dans son ouvrage hâtif, La Fin du monde.

5. The Encyclopedia of Apocalypticism, 3 vol.

6. En particulier dans son album Mechanical Animals (voir « The Last Day on Earth »).

7. Voir le dernier opus du chanteur, The Golden Age of Grotesque.
} 
toile de fond pour la construction du soi. Mais la fin du monde tend à n'être plus interrogée vraiment. Tenue comme évidence, elle est évidée de son mystère, et l'on peut se raccrocher aux prêt-à-penser. Par l'ostension du désastre chrétien durant des siècles, l'apocalyptisme comme description de l'inversion antéchristique, puis de la dernière heure, s'est bien essoufflé. Il demeure les termes de la fin absolue, dont le contenu se projette toujours.

Le millénarisme qui se liait à ces croyances ne cesse de se relancer. En dépit de désaccords sur le compte des années (le bonheur dans ou pour mille ans?), se dit là l'espérance d'un autre monde et la mort de celui-ci. Le royaume du Seigneur terminera cette réalité de douleur, la révolution prolétarienne mettra fin au capitalisme, le Reich de mille ans clora l'univers «enjuivé», la venue des aliens arrêtera le cours de la vie terrestre, etc.

L'eschato-logie, entendue comme tout discours théorique sur la fin (dans et hors de la seule théologie), s'adjoint ici. La mort s'associera quelquefois à celle du monde même, comme l'affirme Jacques Derrida dans l'avant-propos de son récent ouvrage reprenant des hommages et oraisons funèbres: "La mort de l'autre, non seulement mais surtout si on l'aime [...] déclare chaque fois la fin du monde en totalité, la fin de tout monde possible ${ }^{8}$.» Lucrèce bien avant Derrida (et autrement) postulait le dépérissement de la terre et celui du corps humain comme des réalités complémentaires ${ }^{9}$. Cela allait chez lui avec une affirmation de la décadence. Le phénomène (naturel) du déclin ne pouvait pas être enrayé, la planète ne donnerait plus de beaux fruits, tout cesserait. Dans une approche organique des sociétés, Oswald Spengler, au début du $\mathrm{XX}^{\mathrm{e}}$ siècle, parlait du Déclin de l'Occident comme d'un crépuscule inéluctable (le bout sera atteint vers 2220) ${ }^{10}$. Il y aura à distinguer tout à l'heure avec d'autres interprétations partisanes de la décadence, qui promettent la fin du monde «à moins que... ${ }^{11}$ » - qui, donc, annoncent et réfutent l'apocalypse politique.

Dans tous ces cas, le prophétisme paraît de mise, ce qui n'est sans doute pas si obligatoire. Avec Jean de Patmos, comme chez Daniel (Dn 11-

8. Chaque fois unique, la fin du monde, p. 9. Publié en 2003.

9. De rerum natura, II (fin).

10. Der Untergang des Abendlandes, vol. I, voir tableau n ${ }^{\circ} 3$.

11. Charles Maurras, L'Avenir de l'intelligence, p. 153. 
12), la fin est vue en avance, et les hommes sont avertis. Quand une date précise est délivrée pour le dénouement et qu'il ne se passe pas, cela fait problème. Une étude sociologique assez ancienne avança que les sectes millénaristes, et démenties «par les faits», n'avaient alors plus qu'à récolter les fruits de leur inexactitude, que la non-fin renforçait au contraire la foi des croyants si le groupe des adeptes était suffisamment structuré ${ }^{12}$. Cela se vérifie partiellement pour les grands monothéismes (mais leur calendrier est flou), cela reste à voir pour d'autres cultes, qui auraient tendance à se dissoudre ou se consumer, bref à détruire leur monde religieux ${ }^{13}$. Le prophète est bien un homme de la catastrophe. Le Déluge, et ses traditions si anciennes, est aussi une fin du monde, préparée quelque peu, quoique fondamentalement brutale. Le catastrophisme est autant à l'œuvre dans ceux, rares, qui promettent l'éternité de la fin du monde.

Ces positions ont été largement illustrées par la science-fiction. Je veux dire davantage. Dans la genèse de sa constitution moderne, durant les années 1880-1890, ce qui s'appellera la SF présente le futur comme la fin du monde. Chez Camille Flammarion, notre planète s'éteint (et renaît après sa fin); pour le narrateur du Horla de Maupassant, le monde humain se perd dans «un bûcher monstrueux, éclairant toute la terre $^{14} \gg ;$ L'éve future de Villiers de 1'Isle-Adam est la créature artificielle qui rendrait peut-être un monde perdu ; La Machine à explorer le temps de Wells conduit son explorateur dans les temps des fins. Le souvenir de ces livres hante même l'optimisme progressiste de sciencefiction et rejaillit dans toutes les proclamations de fin d'univers (dont l'inévitable Matrix, qui tue le réel en plusieurs coups).

- Apocalypse, millenium, eskhaton, décadence, déclin, dégénérescence, prophéties, oracles, prédictions, catastrophe, futur... conduisent par un réseau de routes et d'impasses aux limites de notre monde. Les discours s'organisent et doivent être destitués si l'on se donne la chance (le risque) d'aller à la fin.

Le Langage oublié, le dernier album du chanteur Gérard Manset, est presque organisé entièrement autour de la fin du monde. Les dix

12. Leon Festinger et al., L'Échec d'une prophétie, p. 2-5 surtout.

13. La dissolution de la secte étudiée par Festinger n'est peut-être pas si incidente que cela est dit (voir conclusion). Les disciples «aumistes», du Temple solaire ou de D. Koresh ont provoqué plus ou moins directement leur propre liquidation.

14. Guy de Maupassant, p. 938. La venue du Horla dit la fin du monde humain. 
chansons seraient ainsi dix façons de désigner cet événement, sans volonté dogmatique, en gardant au contraire un écart entre des approches non unifiées du problème. «Demain il fera nuit» use du prophétisme apocalyptique, et le revendique («Je l'ai lu dans un livre $\gg)^{15}$. Une pensée interrompue revient successivement aux traditions usées pour faire sourdre une vérité malgré tout. Les rivages marins depuis quoi Lucrèce contemplait le naufrage de la vie humaine (avec le célèbre suave mari magno ${ }^{16}$ ) deviennent par exemple «le bord de l'étang»d'où l'«on voit la fin du dernier monde connu». Manset le rhapsode chante à partir des pensers anciens, il mêle le futur des «enfants mutants» du «XXI ${ }^{\text {e }}$ siècle» aux rêves d'un avenir loin du «mensonge aux foules» de la politique, la prédiction catastrophique («Et les rats s'enfuiront») à l'évocation peut-être d'un paradis perdu et retrouvé («Aujourd'hui c'est hier, hier c'était demain»); il continue. Il délivre dix morceaux de la fin. Le «langage oublié» est en résurgence chez lui, il revient altéré pour proclamer la fin, la sienne, celle du monde, et celle des normes qui faisaient mine de la dire. Mais il n'y a pas une conception une.

Pour faire saillir l'écart, je citerai le Villa Vortex de Maurice G. Dantec. Son roman au contraire participe de la fiction totalisante, dont Lovecraft fut un promoteur. Tout fait sens en tout. La première partie de l'ouvrage n'est pas pour rien une enquête policière, où des indices épars sont les marques d'un dessein général. De même, les types de paroles sur la fin sont connectées dans une machinerie de la destruction. Il ne s'agit ni d'une réédition sans changements (traditionalisme), ni d'un recours sémiotique (imagerie), ni d'une juxtaposition singulière (discontinuité signifiante, Manset), il y va d'une ré-interprétation globale, qui, en cela, copie et dérègle un mouvement de mondialisation (comme l'ADN, cher à Dantec, réplique et se trompe parfois).

\section{La mort d'un ami}

«Chaque fois unique, la fin du monde », suggère Jacques Derrida ${ }^{17}$. Ou autrement: qu'il y ait $l a$ fin $d u$ monde n'implique pas la disparition

15. Les citations de Manset sont tirées du Langage oublié.

16. De rerum natura, II (début).

17. Titre même de son livre précédemment cité ; voir son « avant-propos ». 
automatique de la fin, et du monde. Des mondes se sont achevés qui connurent cet événement impossible à répéter et qui se renouvelle pourtant. Encore doit-on penser que la fin est susceptible de se proclamer et de différer de soi. Même la disparition de ce système solaire n'est pas celle de l'univers, ainsi de suite. Et «quand on perd un ami» (Manset), le monde s'écroule derechef. La mort de l'un pourrait précipiter celle de tous en soi-même, ce qu'énonce Derrida dans la préface d'oraisons funèbres qu'il écrivit. Pourtant, toute fin du monde ne se ramène pas à la mort de l'autre. Cette catastrophe mondiale est aussi le décès, elle ne s'y résume pas.

Plusieurs mondes se prétendent le monde. L'expérience déjoue l'identité comme principe. Ce n'est jamais que ce monde-ci qui s'engloutit (notre planète, notre civilisation, notre temps, notre espoir, notre vie, notre nôtre), et pourtant le monde absolument un et défait s'éteint. Ce pourquoi distinguer la fin $d$ ' $u n$ monde et celle $d u$ monde n'a aucune valeur. Les eschatologies ont souvent besoin d'une assertion univoque et dangereuse (le c'est nous qui fonde la tribu, la nation, l'ethnie, la race, le pays, la communauté...). Dès qu'elles se mettent toutefois à penser la fin, elles sont conduites à réviser leur positivité même.

Quand Manset rejoue dix fois différemment au moins l'apocalypse terminale, il tient «ce qui ne se peut pas», ce monde et ce monde et ce monde. Les jours d'énervement, on pourrait en déduire que tout cela ne vaut pas, qu'à dire contradictoirement, on ne dit rien de la fin. $\mathrm{Ou}$ au contraire, cette multiplication construit la singularité. Il se pourrait, oui, que ce ne soit pas ça ou ça la fin du monde, mais qu'elle ait lieu.

Le phénomène a-t-il une urgence aujourd'hui qui justifie de s'y attarder? S'il y eut d'autres fins, la fin en est-elle une, ou ne s'en donnet-il qu'une, terminale ? Après tout, «la fin du dernier monde aimé », «la fin du dernier monde possible», Manset n'en faisait-il pas depuis si longtemps son sujet? Alors on répondrait triplement. Oui cela se fit auparavant ; mais cela n'empêcherait pas l'inouï de la douleur maintenant; puis les mondes se refont de manières si différentes qu'il est des époques où un rien les jette dans le rien. Ce qui vaudrait pour d'autres.

\section{Worlds}

La mondialisation encore désigne l'infini devenir-monde du monde. Ce qui n'interdit pas les particularités du devenir que nous traversons. 
L'une d'elles pourrait être ce désir exténué de communication, faire que tous les mondes se disant le monde édifient le monde pluriel. C'est un cauchemar qui légitime à rebours «le choc des civilisations » de Samuel Huntington, qui se contente de nous servir du Spengler à la sauce Guerre des mondes. L'erreur commune est de croire que les mondes sont exactement coextensifs : les bellicistes l'admettent quand ils inventent des affrontements entre sphères, de même que les pluralistes dogmatiques qui parlent de juxtaposition harmonieuse. Je suis en ce monde-ci et seulement, et dans cet autre et uniquement. Mes mondes comme moi peuvent mourir plus d'une fois. Le nous du monde ne nécessite pas la disjonction (us or them), malgré l'historique des confusions sans nombre, qui se poursuivent. Le nous n'est pas ethnocentrique ou raciste par nature. Affirmatif par delà sa négation, et donc conjonctif, il renvoie à l'expérience d'être autre et soi.

La mondialisation contemporaine (qui excède largement la politique ou l'économie) fait craindre que plus de mondes disparaissent que d'ordinaire. Je doute que la perspective d'autre chose (super-capitalisme, alter-communisme ${ }^{18}$ ) suffise à nous consoler de ces pertes, du moment qu'elles s'avéreraient. Une peur s'exprime, définitivement: que le dogme de la coextensivité vise à éradiquer l'existence même du monde. On aurait un choix contraint. Soit la lutte à mort impliquant absolument tout le monde: les membres de chaque «civilisation» devraient faire bloc, et réaliser culturellement cet Einheitsfront racial que réclamait Hitler dans Mein Kampf ${ }^{19}$ afin de se libérer des oppresseurs antagonistes. Soit pour chaque monde, l'interdiction de sa reconnaissance effective, au nom du pluralisme, puis son intégration dans l'uniformité «respectueuse», ramenant la différence à des éléments définitoires fixés par le grand manitou consensuel: assassinat des cultures minoritaires dans un arraisonnement patrimonial, obligation du métissage (n'est-ce pas aussi ce que firent les Blancs esclavagistes?), etc.

Pour les deux cas, dans le futur plus qu'auparavant, la mort d'un monde se propagerait aux autres, ou ce qu'il reste. Partant, des mondes

18. «Alter-communisme» se réfère au mot «communisme» qui clôt le livre altermondialiste de Michael Hardt et Toni Negri, Empire.

19. Adolf Hitler, p. 777. 
qui communiquaient mal (ou pas) communieraient dans une dévastation synchrone.

- Le relativiste demande: est-ce si net?

\section{L'incarnation politique}

Que le discours d'une fin du monde ne conduit pas nécessairement à une politique repoussante.

La décadence, le déclin, la dégénérescence ne font pas très envie, il paraît qu'ils sentent mauvais; les paroles qui les déplorent ont la même odeur. Une «société en décomposition» sur un «lit de pourpre et de fumier», clame l'antisémite Édouard Drumont en 1889 dans $L a$ Fin d' un monde ${ }^{20}$. Face à «la bolchevisation juive du monde», Hitler en appelle à la «haine contre ceux qui pourrissent le peuple et la patrie ${ }^{21}$. L'Occident de Huntington devrait remédier au «pourrissement interne accélérant son déclin ${ }^{22} »$. Et Trotski souhaite la révolution pour échapper à la «catastrophe» qu'amène le «capitalisme pourrissant $»^{23}$.

On peut y voir une rhétorique, on force le trait de la décadence pour rendre plus urgent un sursaut. La relation entre ces motifs et l'action politique pourrait être moins contingente qu'il n'y paraît. Mais de toute façon, les politiques de réaction au déclin sont amenées à reprendre l'imminence. Attention, nous sommes au bord du gouffre - «à moins que...» la Contre-Révolution, l'antisémitisme, l'Extermination, la guerre, j'en passe. «Tout est possible; mais rien n'est inévitable ${ }^{24}$.» L'espoir d'un rétablissement in extremis oriente l'analyse. On évitera cette mauvaise mort totale pour mieux installer le nouveau monde (New world order, Reich, communisme...).

Toutefois, lutter politiquement contre la fin si c'était vraiment la fin maintenant - si l'on ne se contentait pas là d'agiter l'épouvantail rhétorique -, s'adonner à l'élaboration de la meilleure Cité à venir quand on dit qu'il n'y a plus rien, qu'est-ce que cela pourrait signifier?

On voit bien la logique des premiers chrétiens. Quand, dans l'épître aux Romains, Paul encourage la soumission aux pouvoirs établis, il

20. P. 1.

21. Adolf Hitler, Mein Kampf, p. 752 et 780 ; je traduis.

22. Samuel Huntington, Le Choc des civilisations, p. 336.

23. Léon Trotski, Programme de transition, chapitres 1 et 2, p. 17 et 19.

24. Samuel Huntington, Idem, p. 335. 
ajoute rapidement que le salut est de plus en plus proche de toute façon (Ro 13, 1-8 et 11): la politique des hommes va tomber avec leur règne, inutile de s'attarder aux choses publiques. Sous la forme laïque marxiste, cette structure eschatologique ne prône évidemment pas le renoncement. La fin de ce monde implique la disparition de ses moyens politiques traditionnels (ceux de la «démocratie formelle»). L'activisme du révolutionnaire est légitimé par cet effacement prévu, car il relève à la fois d'un univers périssable et du futur socialisme réel. Par là, Trotski, que je citais plus haut, se sépare des autres orateurs de la pourriture, qui souhaitaient prévenir, voire guérir l'agonie. L'œuvre politique du marxiste en un temps de déclin n'est qu'une préparation mortelle à son action post-révolutionnaire. Comment, entre l'avant et l'après de l'insurrection, les deux pratiques se lieraient-elles? par quel miracle? et pourquoi le monde communiste, fût-il établi, serait-il éternel à son tour? On sait que ces questions insolubles expliquent une partie de l'échec. Nous faut-il croire encore qu'une pratique politique liée au monde qui disparaît puisse se transmuer ensuite, par alchimie, palingénésie? Quoi qu'il en soit, nulle fin effective et sue ne devrait strictement justifier la réaction, dont les oracles extrémistes ne sont au fond qu'une manipulation mensongère ${ }^{25}$.

\section{Le sentiment - La fin}

Trouver dans le sentiment la force de la pensée. Et si la fin du monde ne se vivait pas dans l'explication, s'il fallait la ressentir? Puisque les mondes ne sont pas coextensifs, on comprendrait mieux que tel ami ne voie rien, et s'insurge contre la fin - quand je ne sentirais que cela. Notre univers d'amitié est-il moins menacé?

Le sentiment n'est pas le moins réel, quoique pas le rationnel. Ni le mythe, quoique pas l'irrationnel. Sentir, exister, se transporter.

Il y eut des fins de mondes, qui le niera? Ils sont nombreux, ceux qui firent mine d'en annoncer une. Mais vivre la fin aurait lieu sur le mode sentimental seulement; au risque de connaître vraiment la mort qu'on se crée et s'apercevoir ensuite qu'on aurait pu se suicider diffé-

25. Cela vaut pour Maurice G. Dantec - apparemment fatigué de lire ses propres romans sur la disparition inéluctable et absolue -, pour sa malheureuse marionnette félicitant sur le web la «jeunesse identitaire » d'organiser un combat bloc contre bloc. 
remment. Pour froidement constater le décès, il faudrait être sans rapport avec le mort, ou avoir traversé le trépas de part en part, qui sait?

Ou bien: l'époque de notre monde, son épokhè est plus qu'une suspension du temps, la cessation. Un moment indéterminé qui instaure une fin absolue, pas un lent déclin, un terme complet - mais qui dure? Les chroniques recrudescentes s'écrivant chaque jour, histoires, chants, fictions, poèmes, narrations, philosophies, installeraient-elles l'état chronique de notre fin comme événement?

Peut-être qu'un des mondes où nous sommes, peut-être que notre monde ne se trouve plus que dans le sentiment de sa fin?

\section{RÉFÉRENCES}

Livres

Boia Lucian, La Fin du monde. Une histoire sans fin, Paris, La Découverte, 1989.

DANTEC Maurice G., Villa Vortex, Paris, Gallimard, 2003.

DERrIDA Jacques, Chaque fois unique, la fin du monde, Paris, Galilée, 2003.

Drumont Édouard, La Fin d'un monde. Étude psychologique et sociale, Paris, Savine, 1889.

Dumas-ReungoAt Christine, La Fin du monde. Enquête sur l'origine du mythe, Paris, Les Belles-Lettres, 2001.

The Encyclopedia of Apocalypticism, dirigée par Stephen J. Stein, New York, Continuum, 1999, 3 vol.

Festinger Leon, RieKen Henry W., Schachter Stanley, L'Échec d' une prophétie. Psychologie sociale d'un groupe de fidèles qui prédisaient la fin du monde, Paris, Puf, 1993.

Firolamo Giovanni, Millenario e New Age. Apocalisse e religiosità alternativa, Bari, Dedalo, 1999.

Flammarion Camille, La Fin du monde, Paris, Flammarion, 1894.

HARDT Michael, Negri Toni, Empire, Paris, Exils, 2000.

HitLER Adolf, Mein Kampf, Munich, Zentralverlag des NSDAP, 1939 [rééd.].

Huntington Samuel, Le Choc des civilisations, Paris, Jacob, 1997.

LuCRÈCE, De rerum natura, Paris, Flammarion, 1997. 
Maupassant Guy de, Le Horla, dans Contes et nouvelles vol. II, Paris, Gallimard, 1979.

MaurRas Charles, L'Avenir de l'intelligence, dans Euvres capitales, vol. II, Paris, Flammarion, 1954.

SPENGLER Oswald, Der Untergang des Abendlandes. Umrisse einer Morphologie der Weltgeschichte, vol. I, Munich, Beck, 1920.

Stoicorum veterum fragmenta, vol. II, texte grec et latin édité par Joachim von Arnim, Stuttgart, Teubner, 1903.

Trotski Léon, Programme de transition. L'Agonie du capitalisme et les tâches de la IVe Internationale, Paris, La Brêche, 1983.

Weber Eugen, Apocalypses et millénaires. Prophéties, cultes et croyances millénaristes à travers les âges, Paris, Fayard, 1999.

Disques et flux

DANTEC Maurice G., lettres adressées au Bloc identitaire, http://www.bloc-identitaire.com, 2004.

MANSET Gérard, Le Langage oublié, EMI France, 2004.

MANson Marylin, Mechanical Animals, Nothing/Interscope Records, 1998.

-, The Golden Age of Grotesque, Nothing/Interscope Records, 2003. 